Mathematical \& Computational Applications, Vol. 8, No. 3, pp. 361-368, 2003

(c) Association for Scientific Research

\title{
SIMULATION OF SWARM INTELLIGENCE AND POSSIBLE APPLICATIONS IN ENGINEERING
}

\author{
Savaş Öztürk and E. Murat Esin \\ Gebze Institute of Technology, Faculty of Engineering \\ Gebze-Kocaeli / Turkey \\ ozturk@btae.mam.gov.tr, emesin@bilmuh.gyte.edu.tr
}

\begin{abstract}
Modeling biological and natural systems in order to solve complex problems have become popular. Traditional techniques fail at solving some types of problems. On the other hand, it is seen that these kind of problems are solved in nature without help of human. Swarm intelligence(SI) as a research field, proposes such solutions. SI models the collective behavior of the social insects like ants, bees or termites and their coordination without communication. The emerged intelligence has some special characteristics and it can be applied to some areas. Some of the application fields are robotics, networking, finance and entertainment.

In this paper, principles of SI systems, original simulations of some SI techniques, and new approaches are presented.
\end{abstract}

Keywords - Swarm intelligence, clustering, pheromone, agent

\section{INTRODUCTION}

Ants, bees and even bacteria are amongst the many organisms that display sophisticated kinds of cooperative behaviour as a survival strategy. Cooperating for a single task such as foraging for food can be much more efficient than letting each individual fend for itself. Scientists and engineers who seek efficient solutions to complex tasks are learning valuable lessons from insects [1].

Benefits of an individual is more important than social benefits from the view of mankind. According to social insects, working for the good of colony is the most important principle. Ants are a classical example of social insects and they work together for the colony.

Biological research on ants has shown that an individual ant has very simple characteristics and it is nothing without being a member of a colony. But the collective work of them emerge an extraordinary synergy. Swarm intelligence is defined as "the emergent collective intelligence of groups of simple agents." [4]. For example ants find the shortest way to the food from nest, termites cluster dead and larvas in order to clear the environment, wasps build strong nests and so on. Finding the shortest way is applied to the Travelling Salesman Problem, an NP Hard problem. Clustering the dead is applied to finance and banking in order to cluster client files. Nest building, cooperative transportation and division of labour of social insects are tried to be modeled. It is able to show more applications and ongoing projects.

It is important to learn the characteristics of social insect behaviour exactly. Biologists try to discover the enigma behind the cooperative actions of social insects. In fact, applications of biological and natural systems sometimes fail and research seems not to be completed yet. Ants and termites which are classic examples of social insects 
are examined with two of their popular techniques. First one is foraging behaviour of ants and second is clustering behaviour of termites. Two simulations are developed the processes of both properties.

\section{CHARACTERISTICS OF SOCIAL INSECTS}

It is important to understand the characteristics of social insects in order to model them exactly. Especially ants are classical examples for SI. For this reason, it is useful to determine biological and behavioral properties of ants.

Ants are modeled for several disciplines.For example; they are known as hard-working and economical insects and they are modeled by economists. Architectures try to model their nest building task. Ants live in colonies and they have important mechanisms as listed below:

- cooperation and division of labor

- adaptive task allocation

- work stimulation by cultivation

- pheromones

Ants are interesting because they solve complex problems by local means, their productivity is better than their single activities. If some members fail to operate their job, the complete system won't be effected. Anst are the grandmasters in search and exploitation.

The analogies between information systems and social insect characteristics have been interesting for researchers. Distributed sytems of interacting autonomus agents, self-organized control and decentralized cooperation, division of labour and distributed task allocation, indirect interactions, and trying to obtain performance optimization and robustness are some of the similarities.

\section{SIMULATION OF SI TECHNIQUES}

\subsection{Finding The Shortest Way (Foraging Ants)}

Recent research on ants has shown that ants are able to find the biggest food source besides they can find the shortest way to there. They don't have a measurement control, they don't have a distance sensor. Also they all have their own agendas and they have different jobs to do. For example, some of ants collect food, some of them feed hungry ones, some of them are responsible for cleaning dead and grouping larvas. But they interact perfectly, and they are able to find the shortest way. An individual ant has not a capability of finding the shortest way, it can walk randomly and has some simple rules according to its job. They don't wait, they don't have time for thinking or discussing. There is no commander, the queen ant is only responsible for reproduction and it stays in the nest. The answer of the question "How can they succeed at finding the shortest way?" lays in the biological characteristics of them. Ants exude a chemical substance named pheromone that attracts the other ants. Ants have a simple mechanism to follow the trails of pheromone while walking. Pheromone trail evaporates in a period of time and the effect of pheromone depends on the amount of it. 
According to Figure 1 it is assumed that there are two ways between the nest and the food source, one is short and the other is long. Ants begin to leave the nest to find the food and bring to the nest. They walk randomly and it is assumed that same number of ants prefer the two ways two go at first. It is known that all the ants exude pheromone. The ants which go from the short way come back earlier to the nest and they leave two units of pheromone while other ants leave one unit. When the recent leaving ants arrive to intersection of two ways, they sense the amount of the pheromone and they prefer the short way because it has more pheromone. After a time, all the ants prefer the shortest way.

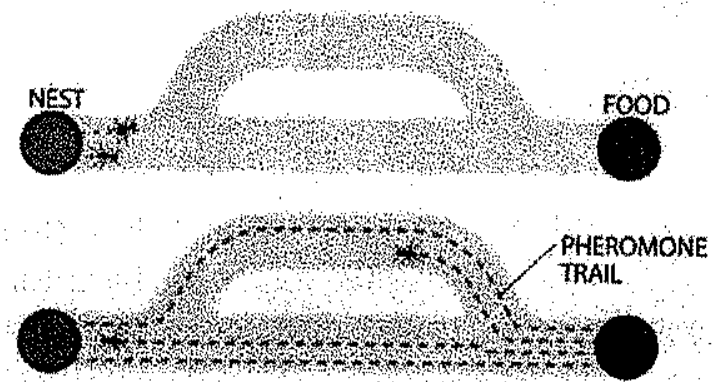

Figure 1. Foraging ant behaviour (Bonabeau et al, 2000)

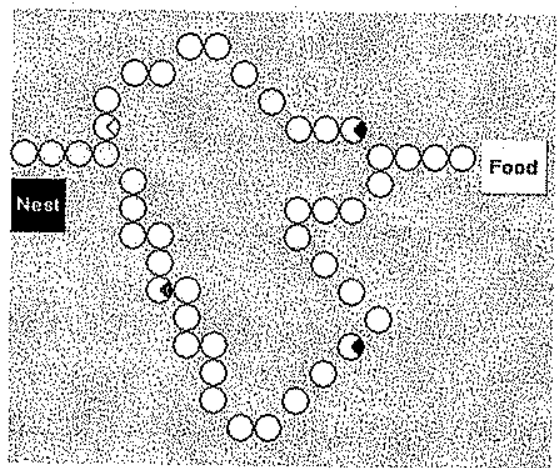

a) Initial state

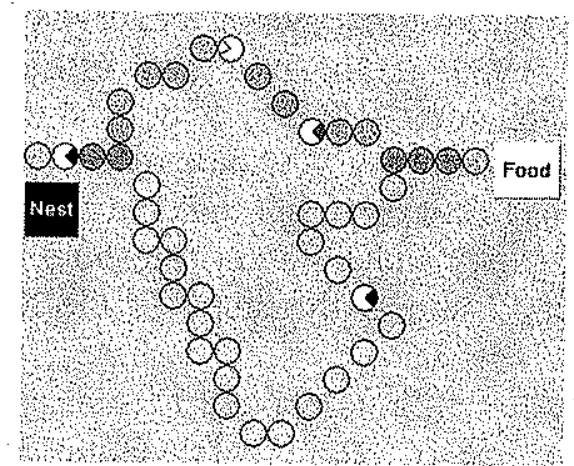

c) Third ant chooses the other way

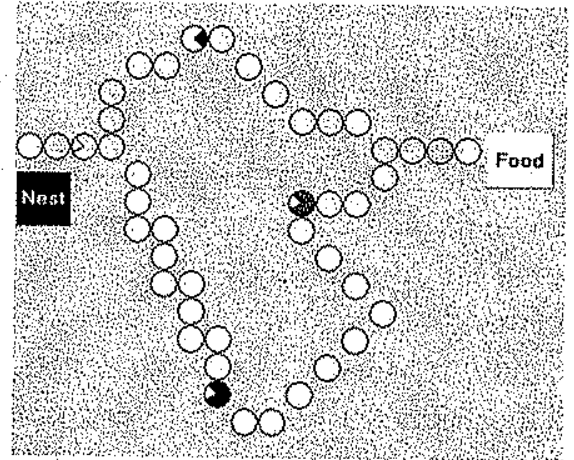

b) Pheromone increases

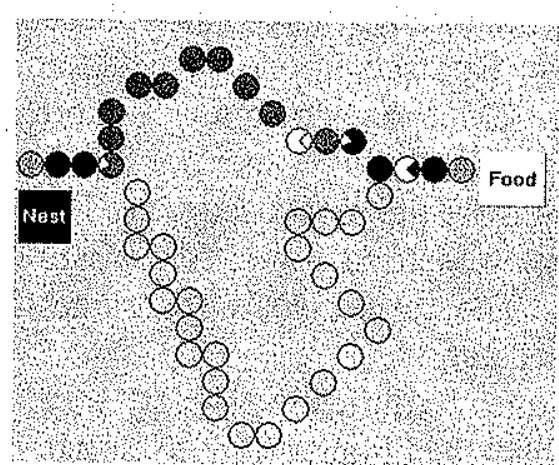

d) All ants are at the same way

Figure 2. Ant foraging simulator interfaces 
Algorithm of finding the shortest route is written below:

Procedure find_the_shortest_way

While there is food somewhere

Random walk

Leave one unit pheromone

Smell for pheromone on the way

If pheromone_threshold_level $<$ pheromone_of_the_way and

pheromone_of_the_way is more than others, go on that way.

Else go on random walk.

If encountered food, take it.

If encountered nest, leave the food.

Wend

Pheromone_of the_way $=$ Number_of_ants_passed $* 16-($ total_steps $/ 10000) * 8$

38 steps are needed to go to the food source and come back for the short way.

66 steps are needed to go to the food source and come back for the long way.

If total ant number is 8 , one tour is completed at $4 *(38+66)=872$ steps.

Simulation program interface coded at $\mathrm{C}++$ is designed to show the demonstration results clearly. It is for demonstration in order to see how the pheromone on the ways changes and how the ants choose the shortest way. First way, shorter way, has 11 units where longer way consists of 25 units. Number of ants and pheromone threshold level are entered by the user. Maximum 8 ants can be chosen as number of ants. Pheromone threshold level indicates when ants begin to sense there exists pheromone on the way. Until this level is not obtained, ants go on random walk.

Ants go to food source on their way and come back using the same way until pheromone threshold level is not exceeded. The default threshold level is 20 for both of the ways. This means that if that way is not passed 20 times by ants, it can not be sensed by the ants. The ants which go to food from shorter way come to nest earlier than the others: Therefore, that way is marked with two units of pheromone where the other way is marked with one unit pheromone, because the ants of longer way has not returned yet. This scenario is depicted at Figure 1. Pheromone amount of one way is shown as different tones of gray. If there is no pheromone on the way its color is near white. If pheromone level increases, the color of that way will be darker. The darker toned way will be the most used way. This means that it is shorter than the other.

The simulation is tried for different number of ants and different threshold levels of pheromone. The result is always same. Figure 2 shows the steps of the Simulator running for 8 ants.

This technique, finding the shortest way while foraging, is applied to some problems that traditional techniques fail to solve them. One of them is Travelling Salesman Problem(TSP). Dorigo has been working on TSP and successful results are collected [2]. Also this technique is applicable for network routing algorithms. It has been using at France and British Telecommunication Networks for facilitating network traffic [3]. 


\subsection{Clustering and Grouping (Dead Clustering of Termites)}

Termites and similar insects clean their environment from dead by clustering them. Also clustering larvas is an important issue for them. There is a simple algorithm for this again. Figure 3 shows the clustering algorithm of termites.

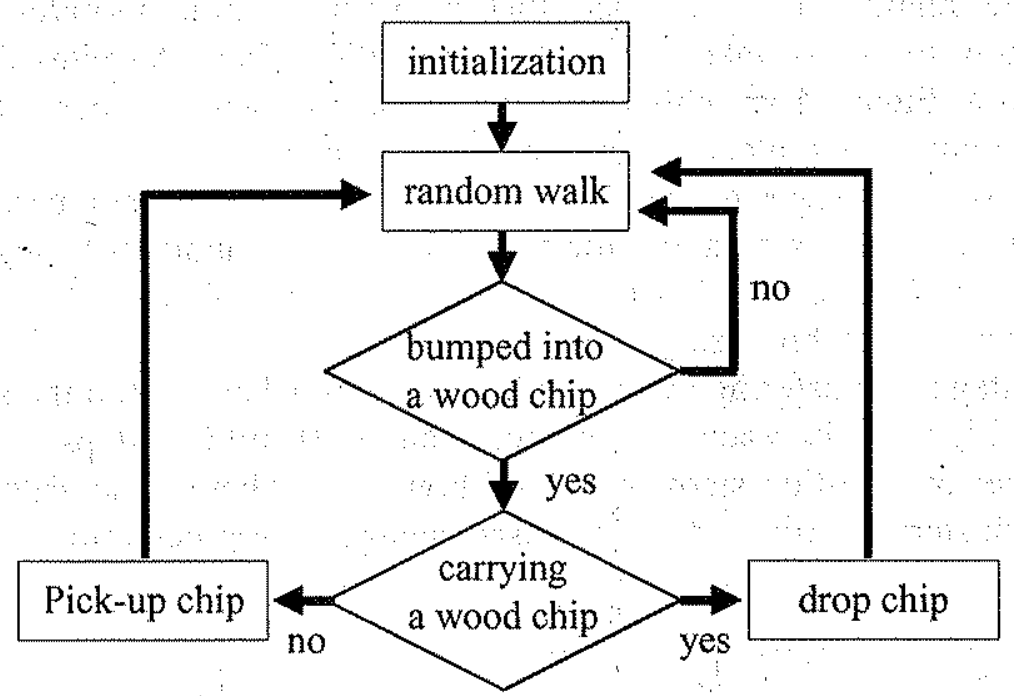

Figure 3. Chip collecting algorithm of termites
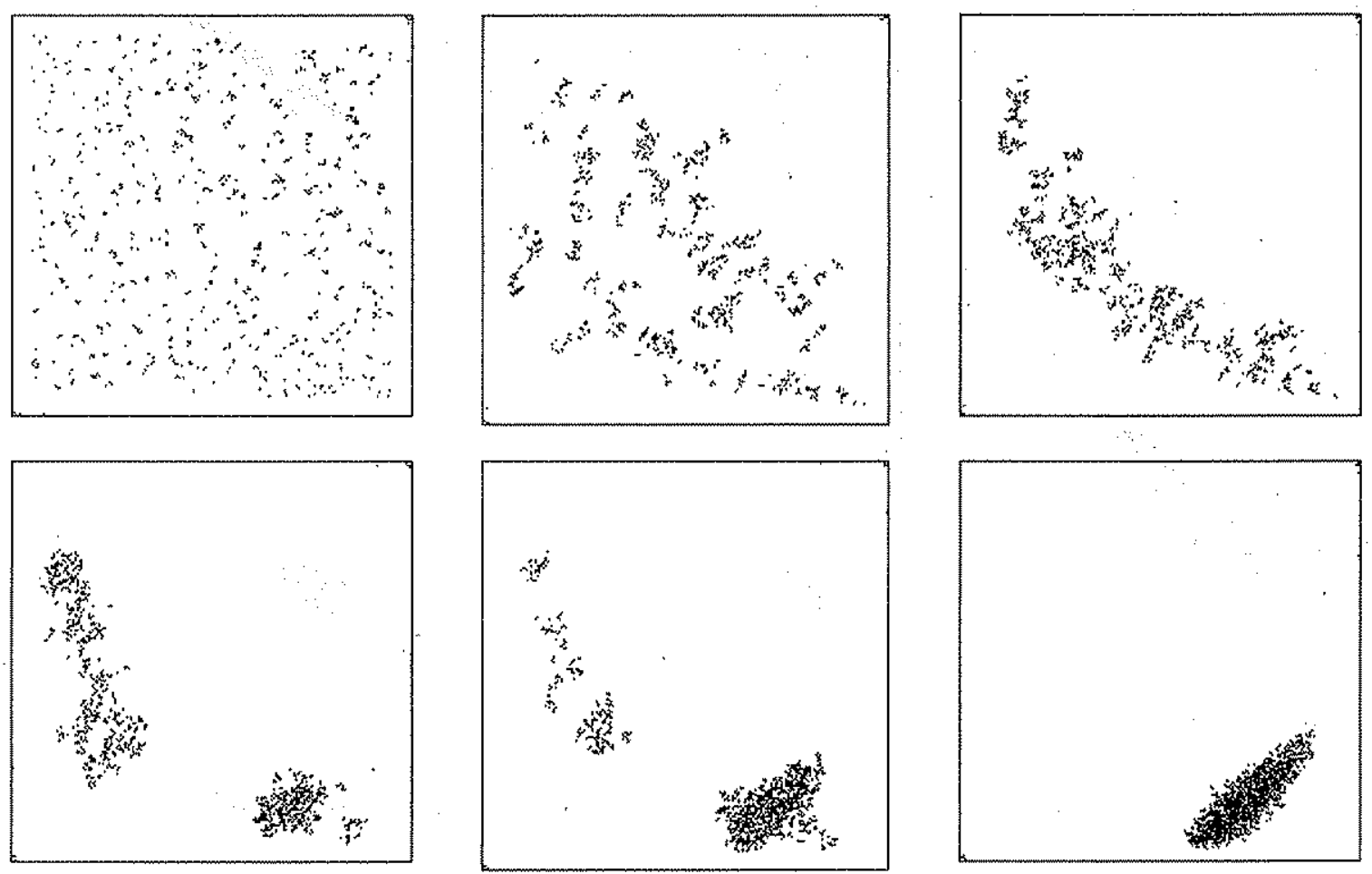

Figure 4. Some views from clustering simulation at different time steps 
A simulation of the algorithm at Figure 3 is coded at $\mathrm{C}++$. A certain amount of virtual termites played their role and clustered the virtual chips. It is important to have a walking algorithm for termites. Termites should go on a way and if they encounter with chip or area border they should return to the opposite direction and continue walking. There are 4 modes of walking positions, and if it is assumed that there is no obstacle on the way the ant behaves as bouncing ball. But, of course, there are a lot of obstacles (1000 chips in this simulation) and termites always changes their directions. To reach the reality, it is assumed that sometimes some termites are died, and sometimes new termites take part in the simulation. Dead termites disappear. Dark pixels shown at Figure 4 are chips, termites are not shown. As seen, after a period of time, all the crumbs are collected, environment is cleaned.

There can be several usages of the resulst of this simulation. A bank used this algorithm to analyse its customers who apply for bank credit. It is important to segment clustered part from all working region. For example, the last view of Figure 4 can be processed by image processing techniques.

The disadvantage of clustering algorithm is estimating when to stop the simulation. The result at the Figure 4 is reached at approximately 10 million steps, but step number could be either one million steps or 100 million steps. There is no arithmetic equation for perfect clustering time. Another disadvantage is segmentation of the clustered region.
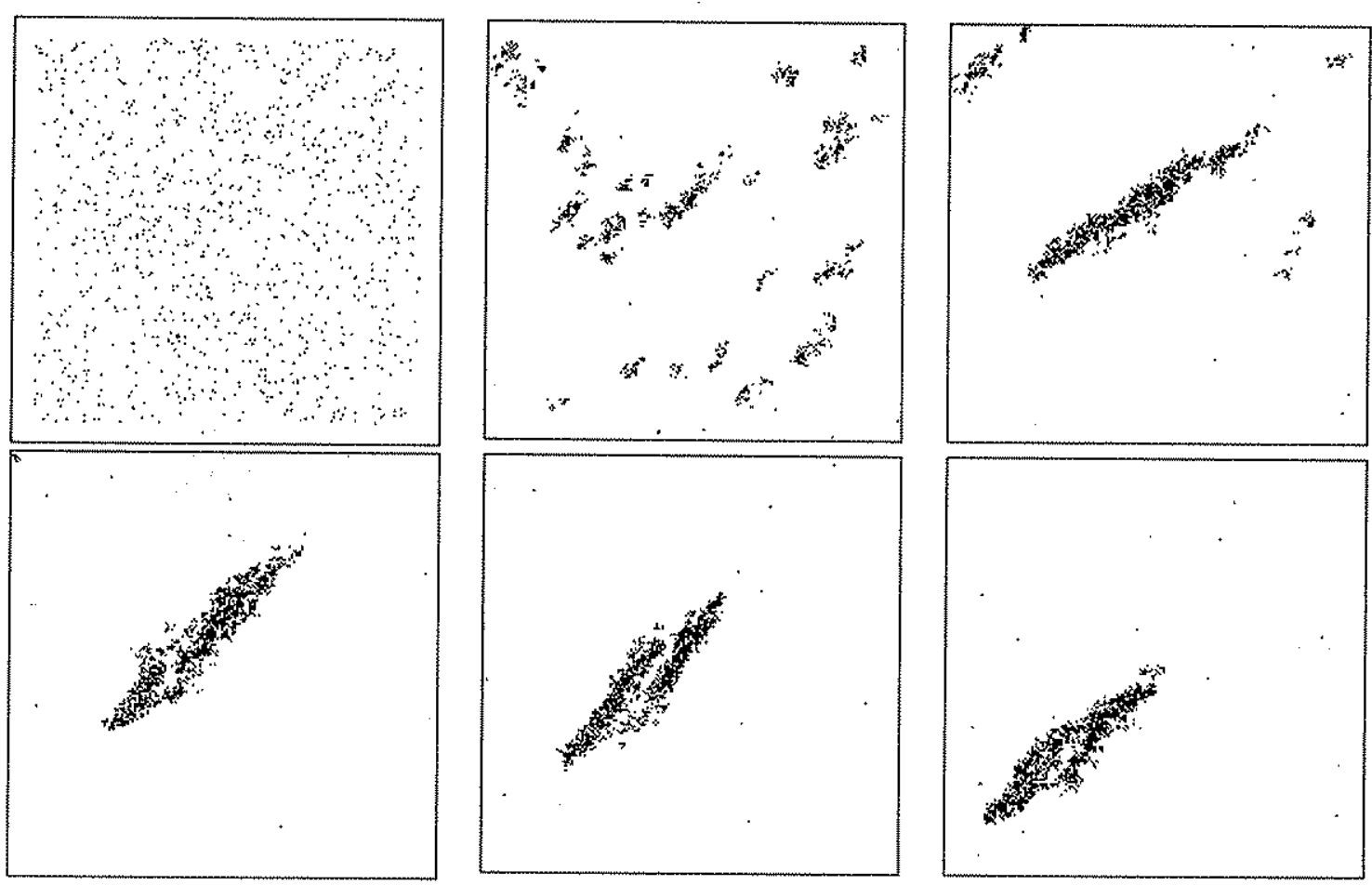

Figure 5. Some views from clustering simulation at different time steps( 2 objects)

Another approach for clustering algortithm is clustering different types of objects. The algorithm at Figure 3 is changed slightly. If the virtual termite encounter one type of object and it carries same kind of object than the algorithm processes as Figure 3 . If 
encountered object is different from carried, the termite only changes its direction, and go on walking. The results are not as successful as the first approach's and shown at Figure 5.

This type of algorithms are useful for clustering data especially for finance and banking. Banks have tones of data that wait to be clustered and analyzed. Behaviour of the termites can be imitated by software agents and can be let to run on database to cluster data. It is noted that malfunction of some of the termites does not effect the operation in any way.

\section{POSSIBLE RESEARCH AREAS}

The simulation programs, which are told in previously, does not require specific techniques, complex solutions or huge amount of memory or disk. These simple algorithms only show an alternative way to solve complex problems. Engineers can apply and adapt these techniques to their problems. But there are problems. It is hard to estimate when to stop the simulation. Also it is hard to completely divide clustered piles into seperate groups while dealing with more types of objects. The simulation algorithms developed at this study must be improved according to new observations on social insects. Although biology can not give absolute data sometimes, this area is worth for research. Some research ideas about SI we propose are below:

- Military Uses: Military units that must collaborate can use the Swarm theory. For example, aircrafts that must to fly together in a group, can obtain the flying stability by using Swarm clustering principle. Here, there must be a robust GPS(Ground Positioning System) structure in order to make the communication strong. Each aircraft is a member of a colony and they follow themselves according to "GPS pheromone" and they cluster.

- Document Management: There are several document management tools for intranet and internet users. It is thought that there can be a system that enable users to find the document which they look for in a quick and efficient way. Users of the system are supposed to be members of the colony and they leave their "digital pheromone" where they visit.

- Education: Advisers need detailed student files while helping troubled students. But most of them usually are not able to be classify and so it becomes hard to keep a good history of the students. Using clustering and grouping technique of termites, files may be organized and clustered well. The agents that mimic termites may map the troubled students easily to the teachers and advisers.

- Banking: Nowadays banks have large databases that they can't analyse them exactly. Using swarm of agents, they can succeed at grouping the client data as they wish. Therefore, they can make stock analysis and other estimations better and identify the debted clients or candidates of debteds easily.

\section{CONCLUSION}

Potential of swarm intelligence is enormous. It offers an alternative way of sytems that have traditionally required centralized control and extensive programming. It 
instead boasts autonomy and self-sufficiency. Systems can be developed they can adapt quickly and rapidly fluctuating conditions. Shortly by simple rules, "complex" behaviour results. But the field is infancy, researchers lack a detailed understanding of the inner mechanisms of social insects.

Especially self organization characteristics of social insects is very convenient for research. Swarm intelligence can be applied easily to military and commercial applications. The Internet, ultimate innovation of this century, can be discovered in depth by the use of swarm systems. To create synergy from the work of unproductive individual parts, swarm intelligence will be known as one of the best ways.

\section{REFERENCES}

Menkov,V., Neu,D.J., Shi,,Q., "AntWorld: A Collaborative Web Search Tool”,

[1] Workshop on Distributed Communities on the Web(DCW 2000), Quebec City, Canada, June 2000

Dorigo,M., Cambardella,L.M., "Ant Colony System:A Cooperative Learning

[2] Approach to "The Travelling Salesman Problem", IEEE Transactions on Evolutionary Computation, Vol.1, No.1, 1997

[3] Bonabeau,E., Theraulaz, G., "Swarm Smarts", Scientific American, March 2000

[4] Bonabeau,E., Dorigo,M., Theraulaz,G., "Swarm Intelligence : From Natural to Artificial Systems",Oxford University Press, 1999 